\title{
Alloimmune and Infectious Complications in Hand/Face Transplantation
}

\author{
Luis Landin • Jorge Bonastre $\cdot$ Pedro Bolado • \\ Cesar Casado-Sanchez
}

Published online: 10 January 2013

C Springer Science + Business Media New York 2013

\begin{abstract}
Vascularized composite allograft transplantation was definitively introduced in the armamentarium of reconstructive surgery in 1998. The development of potent immunosuppressive regimens permitted allograft survival, but rejection and infectious complications remain important issues. Our aim is to provide useful information on immunosuppression, rejection and infectious complications, their clinical management and their repercussion on the functional outcomes and allograft regeneration. Reports that gather current knowledge are included and commented. Current surgical strategies to control perioperative infections in hand and face transplantation are discussed. The implantation of reconstructive transplantation depends much on the control of rejection and infectious complications for the safety of the patients.
\end{abstract}

Keywords Composite tissue allograft - Hand allograft transplant · Complications · Infection - Rejection .

Alloimmune complications · Hand transplantation · Face transplantation - Infectious complications .

Immunosuppression

\section{Introduction}

In 1997 Hofmann et al. [1•] introduced vascularized composite allografts (VCA) to reconstruct large body defects by totally replacing a knee joint using a vascularized knee allograft aided by calcineurin-inhibitor (CNI) cyclosporine

L. Landin $(\bowtie) \cdot$ J. Bonastre · P. Bolado · C. Casado-Sanchez Division of Plastic and Reconstructive Surgery, University Hospital "La Paz", Paseo de la Castellana 261, 28046 Madrid, Spain

e-mail: landinsurgery@gmail.com
A. The lack of knowledge about immunological rejection and the fear of infectious and other complications lead to poor outcomes. However the last 15 years have brought several developments in this field which have exponen-

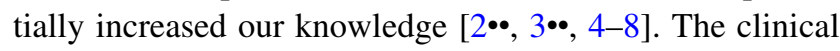
development of VCA transplantation has been headed by nephrologist JM Dubernard, who pioneered the first hand allograft transplantation (HAT) and later the first face

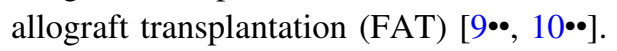

Nowadays hand allografts (HAs) survive and avoid rejection not only under triple standard therapy [9・•], but also under different immunosuppressive (IS) protocols that might deliver fewer complications [11]. Allograft survival is no longer the primary outcome measure after the transplant, and other concerns like the risks derived from IS guide the optimization of the regimens that follow these reconstructive transplantations [11].

Immunological and infectious complications due to the chronic administration of IS drugs are major concerns in these non-life saving procedures [11]. The use of chronic IS and its inherent risks represent the main limitation for the implantation of this field and for the incorporation of solid organ transplant (SOT) developments into the field of reconstructive surgery.

Our aim is to review current trends on immunological and infectious management and their relation with IS and functional outcomes after VCA reconstruction.

\section{Importance of Immunosuppression}

The need for chronic IS after VCA makes the patient medically complex to follow-up management (Fig. 1). It can be argued that VCA transplantation makes hard cases even more difficult, while every effort should be made to 


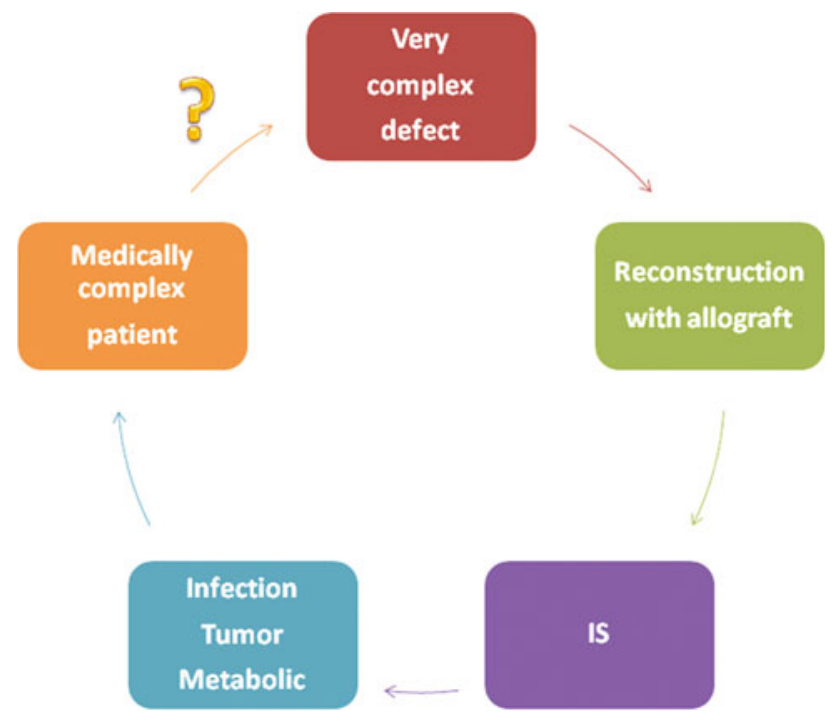

Fig. 1 This figure shows how very large and complex body defects that are reconstructed using VCA need chronic immunosuppression, which exposes the patient to several risks that make the case difficult to manage. A question is raised because such situation does not allow discharging the patient, who is dependent from the surgical team for the rest of the allograft survival

Table 1 Reported complications in 28 HA recipients

\begin{tabular}{lll}
\hline Metabolic & Infectious & Oncological \\
\hline Hyperglycemia (13) & CMV (6) & BCC (1) \\
$\uparrow$ Blood pressure (7) & Cutenous mycosis (4) & MZL (1) \\
$\uparrow$ Serum Cr (5) & HSV (2) & \\
Hyperlipidemia (4) & VVZ (1) & \\
Bilateral hip & EBV (1) & \\
Necrosis (1) & HPV (1) \\
& Osteomyelitis (1) \\
& Diarrhea Cl & \\
& Difficile (1) \\
& Pneumonia (1) \\
& Abscess (1)
\end{tabular}

(n) Number of patients; $\mathrm{Cr}$ creatinine, $C M V$ cytomegalovirus, $H S V$ herpes simplex virus, $V V Z$ varicela zoster virus, $E B V$ Epstein-Barr virus, $H P V$ human papilloma virus, $B C C$ basal cell carcinoma, $M Z L$ marginal zone lymphoma

maintain simplicity and preserve patients' health. Reconstruction of very complex defects using VCA transplantation has been worth it for the patients despite its immunological complications and cost [12]. However, the benefit of VCA transplantation in terms of patient survival and quality of life (QoL) is yet to be measured [13]. Two different reports have been able to demonstrate an improvement in function after HAT as compared to pretransplant basal function using a validated questionnaire $[14 \bullet, 15]$. Specifically, one study performed by our group was able to determine a statistically significant improvement in function in ten recipients of HAs [14•]. However the drawback of such gain was that among 28 patients receiving HAs, there were 52 complications that were attributed to the IS drugs (see Table 1). Fortunately, all the complications could be successfully managed medically or surgically, and none of the recipients requested the removal of the allografts. Despite the reconstruction of the body using allografts may improve body defects, to date the impact of side-effects of IS on the QoL of the recipients remains debatable. Two reports have highlighted the improvement in the QoL after HAT. The fourth report from the Hand Registry found that $75 \%$ of the patients recognized an improvement in their QoL despite the burden of IS $[2 \bullet \cdot$. It also noted a slight trend to a decrease in hand function as reported 2 years after transplantation $[2 \bullet \bullet]$. Another study concluded that most HAT reports showed improvement in the QoL, but more data should be obtained from prospective evaluation assessed with different tools and reported by the patient [13].

To date there is no clear recommendation of which IS regimen carries out the lesser risk of rejection while maximizing the immune capability of the recipient. The level of immunosuppression should be high enough to prevent immunological complications, most feared chronic rejection (CR), but also low enough to avoid infections, metabolic and oncological complications associated to overuse. Scarce information is available since the field is dominated by case-reports and short retrospective series [14•]. Therefore, most recommendations to prevent morbidity due to IS are usually extrapolated from the experience in SOT (i.e. kidney transplantation) [16•].

Efforts are being made to implement variations of IS regimens that may provide a tolerant state to kidney allografts (KAs). Remarkably, Scandling et al. [17•] reported 16 patients that received HLA-matched KAs of which 13 were able to survive without maintenance IS after total lymphoid irradiation and hematopoietic pluripotent $\mathrm{CD} 34^{+}$ cell transplantation from the dono. Another study demonstrated that a regimen consisting of $\mathrm{CD} 34^{+}$transplantation after chemotherapy preconditioning facilitated the survival of five KA in six recipients without maintenance immunosuppression [18]. Currently there is a phase II clinical trial evaluating the safety of a protocol consisting of CD $34^{+}$transplant to facilitate engraftment of HAs in patients under tacrolimus monotherapy after alemtuzumab preconditioning [19, 20]. Such protocol resulted in a number of rejection episodes similar to other triple IS regimen used by other teams, but permitted allograft survival under a single IS drug for the maintenance phase in five recipients of HAS with a minimum follow-up of 2 years [20]. However, it remains unknown whether regimens from SOT looking for tolerance can be directly applied to VCAs, especially with the difficulty to match 
HLA due to the few number of donors and candidates in VCA transplantation.

With regard to recommendation about IS, to date there is no clear recommendation for any IS regimen used during induction or maintenance phases. We evaluated factors influencing acute rejection (AR) of HAs, but were not able to find significant differences between induction regimens with regard to the number of AR episodes during the first semester post-transplantation. Of importance, basiliximab presented a higher number of rejection episodes as compared to anti-thymocyte globulin (ATG) or alemtuzumab, but we were no able to determine if any induction was associated with more complications. We also evaluated if there were any different benefits associated with the maintenance IS, revealing that those recipients under sirolimus-based regimens presented higher occurrence of AR episodes as compared to CNI-based regimens (3.2 vs. $1.9 ; P=.018)$. Unfortunately, we were not able to identify differences with regard to the use of steroids during the maintenance phase (Fig. 2). We were no able to elucidate if any maintenance regimen was associated with higher complication rates, or if there was any association between IS regimens and the occurrence of infection [21•]. Therefore despite variations of IS protocols may provide fine adjustment of IS and minimize their side-effects, only large series of transplants may eventually identify differences which may justify their use [21•]. The same applies to FAT, where series may be even shorter. It seems that most authors performing FAT preferred the safety of standard triple regimen $[10 \bullet \bullet, 22,23 \bullet, 24]$. An exception to this may be the series of three FAT that reported $100 \%$ long-term survival using a steroid-free maintenance regimen [25].
It has been usually defended that IS (i.e. tacrolimus) could enhance the regeneration of the allografts after HAT. This is based on the hypothesis that tacrolimus increases the pathway of GAP-43 protein thus enhancing axonal regeneration observed in experimental models [26]. Only very recently the clinical effect of tacrolimus on nerve regeneration has been challenged in a phase II clinical trial in five patients receiving tacrolimus after nerve primary suturing or reconstruction using autografts. The authors concluded that despite an non-significant increase in the speed of the Tinel sign advancement, the functional outcomes did not exceed those expected [27]. To date, animal models suggest tacrolimus improves neuroregeneration but this is yet to be proved humans.

\section{Importance of Rejection}

AR remains a major concern after hand or face transplantation, and thus many efforts have been made to diagnose, prevent or control AR episodes. Rejection in VCA is mainly detected in the skin, and guidelines for biopsy procurement and histological evaluation are still pending. Despite the ease of clinical suspicion, diagnosis is achieved with the combination of visual inspection and histological evaluation of tissue samples after a minimum of hematoxilin-eosin staining [3••]. Immunohistochemical staining has been used mainly for investigational purposes [3••]. Some teams have used serial biopsies to design different scales for rejection or to elucidate the intricate mechanisms of acute cell rejection [4, 5]. However, elective biopsies may produce some difficulties in the diagnosis and control
Fig. 2 Summary of results: Effect of different variables on HA transplantation. ATG antithymocyte globulin, $C N I$ calcineurin-inhibitor, $C M V$ cytomegalovirus, HLA human leukocyte antigen; open square denotes non-significant differences and filled square denotes statistically significant differences (From Bonastre et al. [21•]; copyright 2012. Reprinted with permission from Wolters Kluwer Health)

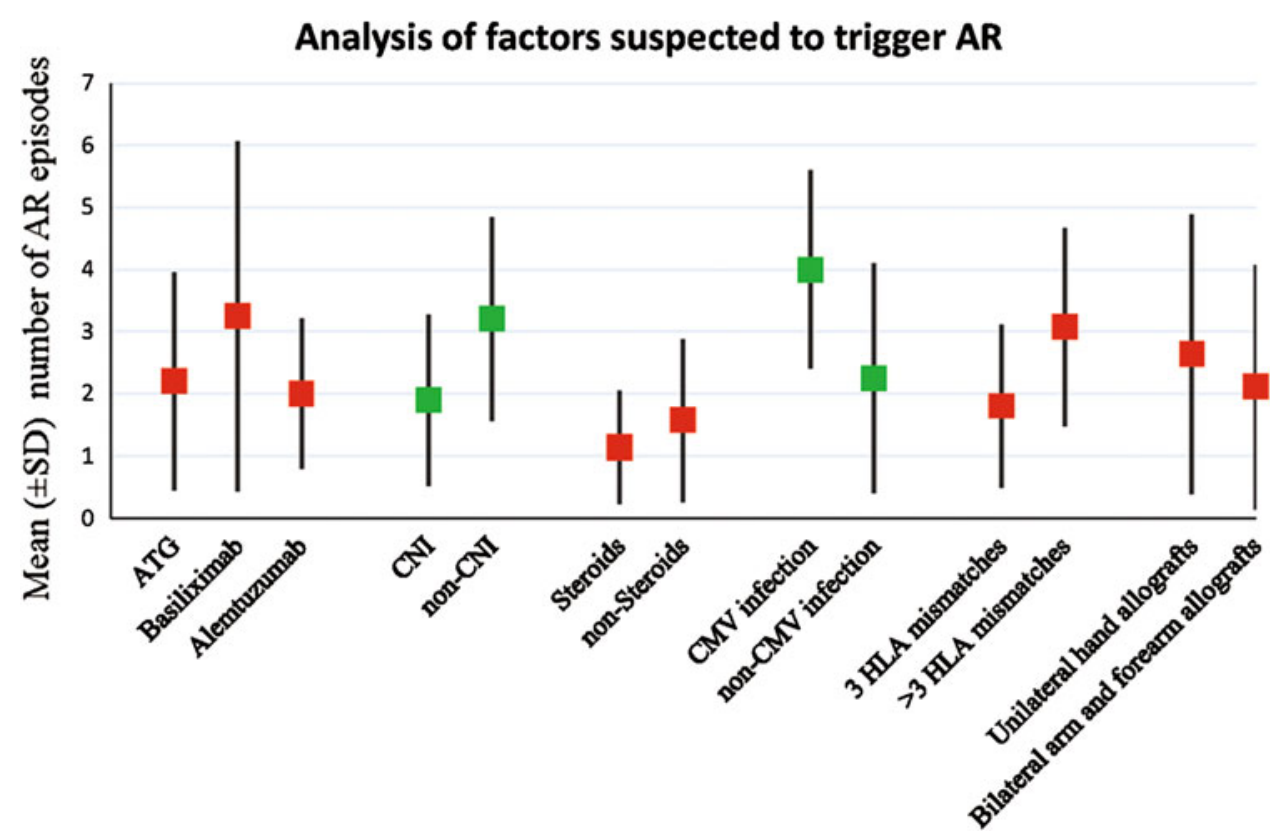


of rejection. For example, clinically rejection-free allografts usually show a low-grade lymphocyte infiltration (i.e. Banff grade I) of the skin [28]. Some authors have even observed histological grade III without clinical signs of rejection at the skin [23•]. It remains unknown if the latter situation requires adjusting of the IS levels, mainly because it has not been demonstrated that lymphocyte infiltration without clinical rejection can lead to CR or graft loss. On the other hand, a few authors have observed a grade I infiltration in very dense and worrying skin lesions [29]. To date most teams prefer to guide their IS therapy by visual follow-up of the allografts [30], but a few have put this strategy into question alleging an unknown risk for $\mathrm{CR}$ $[31,32 \bullet]$.

It is advisable to deal with AR by boosting the IS regimen temporarily, until a new trial for reducing IS is scheduled after a few months. Depletional therapy should be reserved for resistant cases because it carries out a risk of viral infection (i.e. cytomegalovirus, CMV) [7, 8]. Each rejection episode should be accompanied by laboratory determinations like donor specific antibodies (DSA), and CMV ADN counts by polymerase chain reaction because changes in such parameters may trigger rejection [21•]. To date there is no study that clarifies the effect of AR on the function of HAs, but there is a clinical impression that rejection leads to edema and mild functional impairment.

CR has been scarcely described in VCA transplantation. Feature of CR like fibrosis is shown in most biopsied allografts $[22,32 \bullet]$. However only one allograft has been lost and amputated due to chronic ischemia after myointimal proliferation and luminal narrowing [31, 32•]. This patient had received sparing therapy and experienced luminal stenosis due to vasculopathy, and did not respond to percutaneous transluminal dilatation. After removing the ulnar artery it showed severe thickening and it was not possible to revascularize the allograft. Thromboangeitis obliterans was ruled out and the graft had to be removed [32•]. Another patient suffered an impending allograft vasculopathy that was treated with treated with plasmapheresis, high dose intravenous immunoglobulin, and a combination of tacrolimus, sirolimus and steroids, which allowed saving the allograft. These findings prompted the return to triple standard therapy after HAT [32•].

Whether AR leads to CR in clinical VCA transplantation remains unknown, despite it has been remarkably demonstrated in an experimental setting after a serial AR episodes model [33]. It is also unclear whether antibodies play a significant role in rejection of VCAs. Marker of antibody mediated rejection (AMR) $\mathrm{C} 4 \mathrm{~d}$ has been found by a few teams in the allografts, but others were not able to determine [30, 34]. The team from Louisville was able to determine that DSA raised after HA removal due to CR $[31,32 \cdot]$. Further experience and larger series may help clarifying if AMR has a true effect on graft survival or function.

\section{Importance of Infection}

Infections are a workhorse of any transplant procedure and require a close observation of surgical measures intra- and post-operatively. There are great differences in the risks of local infection in HAT as compared to FAT. Local infection in HAT may be due to necrosis of local skin flaps or muscle bellies. Skin flap/muscle necrosis requires urgent debridement and wound stabilization. Depending on the localization, free flap coverage may be used for salvage [34]. Ischemia or congestion of the fingers requires urgent microsurgical revision since it can lead to necrosis and may impair allograft function or even survival [35-37].

Between the second and the tenth day postoperatively local infection after FAT is a major menace for the patient and the allograft and may carry out fatal risks [23•]. This situation requires urgent clinical and radiological evaluation of the neck and thorax preferably by CT-scan. Erythema and edema greatly exceed previous manifestations, and usually the patient presents without fever and under full IS therapy [23•]. Full coverage antibiotics including antifungals are advisable. Surgical exploration should evaluate the mucosal sutures and the retromandibular space, where muscle stumps may have necrosed [24]. If a full FAT (i.e. scalp allograft reconstruction) is underway, the head should be suspended to avoid a decubitus ulcer at the occiput that could necrose and infect the allograft. One main concern during the early postoperative period is the use of the tracheostomy tube, especially if the lower third of the face has been transplanted. Cuffed supraglottic suction-assisted tubes are extremely useful to avoid any risk of aspiration, and the patient can be easily trained on suctioning soon after the operation [24].

In the long-term, infectious events may be similar to SOT, so the patients require a careful preparation, including vaccination before the transplant procedure. The use of vaccines before transplantation should be guided by usual guidelines for SOT. After transplantation, attenuated pathogen vaccines should be avoided. H1N1 vaccination has been recommended during the epidemic in 2010 [16•].

Viral infections should have a special consideration after VCA. Their presentation could be either at the allograft or systemic. Our recent systematic review was able to determine that CMV infection was statistically associated with high number of AR episodes in recipients of HAs [21, $38 \bullet$, 39॰]. Such infection was usually managed with ganciclovir or valganciclovir, but a few required rescue treatments [7]. A similar situation has been observed in a recalcitrant infection due to human papilloma virus (HPV) in the 
allograft of a recipient of $\mathrm{HA}$, that required aggressive treatment due to viral resistance [40]. A repetitive cutaneous varicella zoster infection was also observed in a HA recipient, requiring long-term prophylaxis with acyclovir [30]. Other recipients of FAs have shown non-systemic worrying infection due to herpes simplex virus (HSV) at the lip [23•].

Bacterial infection has been described in HAs in the form of osteomyelitis caused by $S$. aureus, which may be related to hardware implantation. Hematogenous infection of the upper extremity is quite rare, although a few exceptions have been described in immunocompromised children [41]. Radiographic changes are usually absent, but the presence of dead bone should always be suspected. Surgical débridement is critical to the eradication of infection and should be accompanied by prolonged intravenous antibiotic therapy. Wound infection has rarely been observed except from impending allograft failures [36]. Fungal infection has been described in the allograft's skin of two recipients of HAs. One had received high doses of IS due to resistant AR episodes, while the other one was having his therapy adjusted [42, 43]. Both cases responded well to medical treatment. To the best of the authors' knowledge, Pneumocystis jiroveci has not been described in VCA literature. The same holds true for mycobacterial infection in HA recipients, but recipients should screened with the tuberculin skin test. Chemoprophylaxis with isoniazid should be offered if induration is $\geq 5 \mathrm{~mm}$.

\section{Conclusions}

VCA transplantation has been introduced to reconstruct large body defects that could not be managed satisfactorily by other means. However such procedures have a permanent need for IS as their main drawback. There is always a risk of rejection, and medication and surgery expose the recipients to several risks, including infectious diseases. No induction regimen has shown superior results with regard to the occurrence of rejection in the first six months posttransplantation. However, CNI-based regimens have shown superior results as compared to sirolimus-based regimens in the maintenance phase. Clinical inspection usually guides the treatment of AR episodes. The mechanisms and occurrence of CR is yet to be defined in VCA. To date no IS regimen has shown superior results with regard to infection occurrence, though minimization of IS to the degree tolerated by the allograft is preferable. CMV infection has been associated with the occurrence of rejection and donor-to-recipient mismatch should be avoided if possible. Future directions for investigation include the improvement of IS regimens that allow allograft acceptance with fewer complications.
Disclosure No potential conflicts of interest relevant to this article were reported.

\section{References}

Papers of particular interest, published recently, have been highlighted as:

- Of importance

•• Of major importance

1. - Hofmann GO, Kirschner MH, Wagner FD, et al. Allogeneic vascularized transplantation of human femoral diaphyses and total knee joints-first clinical experiences. Transpl Proc. 1998;30(6):2754-61. This paper provides the first description of modern VCA.

2. •• Petruzzo P, Lanzetta M, Dubernard JM, et al. The international registry on hand and composite tissue transplantation. Transplantation. 2010;90(12):1590-4. This report summarizes the world experience in VCA transplantation.

3. - Cendales LC, Kanitakis J, Schneeberger S, et al. The Banff 2007 working classification of skin-containing composite tissue allograft pathology. Am J Transpl. 2008;8(7):1396-400. This paper provides the most widely accepted score of VCA rejection.

4. Kanitakis J, Petruzzo P, Jullien D, et al. Pathological score for the evaluation of allograft rejection in human hand (composite tissue) allotransplantation. Eur J Dermatol. 2005;15(4):235-8.

5. Hautz T, Zelger B, Grahammer J, et al. Molecular markers and targeted therapy of skin rejection in composite tissue allotransplantation. Am J Transpl. 2010;10(5):1200-9.

6. Schneeberger S, Ninkovic $M$, Gabl $M$, et al. First forearm transplantation: outcome at 3 years. Am J Transpl. 2007;7(7): 1753-62.

7. Schneeberger S, Lucchina S, Lanzetta M, et al. Cytomegalovirusrelated complications in human hand transplantation. Transplantation. 2005;80(4):441-7.

8. Schneeberger S, Kreczy A, Brandacher G, et al. Steroid- and ATG-resistant rejection after double forearm transplantation responds to Campath-1H. Am J Transpl. 2004;4(8):1372-4.

9. •- Dubernard JM, Owen E, Herzberg G, et al. Human hand allograft: report on first 6 months. Lancet. 1999;353(9161): 1315-20. This is the first hand transplant reported since the introduction of cyclosporine.

10. • Devauchelle B, Badet L, Lengele B, et al. First human face allograft: early report. Lancet. 2006;368(9531):203-9. This paper reports the first face transplant.

11. Hautz T, Brandacher G, Engelhardt TO, et al. How reconstructive transplantation is different from organ transplantation-and how it is not. Transpl Proc. 2011;43(9):3504-11.

12. Margreiter R, Brandacher G, Ninkovic M, et al. A double-hand transplant can be worth the effort! Transplantation. 2002;74(1): 85-90.

13. Jensen SE, Butt Z, Bill A, et al. Quality of life considerations in upper limb transplantation: review and future directions. J Hand Surg Am. 2012;37(10):2126-35.

14. - Landin L, Bonastre J, Casado-Sanchez C, et al. Outcomes with respect to disabilities of the upper limb after hand allograft transplantation: a systematic review. Transpl Int. 2012;25(4): 424-32. This systematic review shows the first demonstration of functional improvement after hand transplantation as compared to pre-transplantation function.

15. Cavadas PC, Landin L, Thione A, et al. The Spanish experience with hand, forearm, and arm transplantation. Hand Clin. 2011;27(4):443-53, viii. 
16. - Kidney Disease: Improving Global Outcomes (KDIGO) Transplant Work Group. KDIGO clinical practice guideline for the care of kidney transplant recipients. Am J Transpl. 2009;9 Suppl 3:S1-155. This manuscript is a comprehensive review and gives evidence based guidelines for allograft recipient management.

17. - Scandling JD, Busque S, Dejbakhsh-Jones S, et al. Tolerance and withdrawal of immunosuppressive drugs in patients given kidney and hematopoietic cell transplants. Am J Transpl. 2012;12(5):1133-45. This is an important report on clinical tolerance induction.

18. Kawai T, Cosimi AB, Spitzer TR, et al. HLA-mismatched renal transplantation without maintenance immunosuppression. N Engl J Med. 2008;358(4):353-61.

19. Lee WPA. Human upper extremity allotransplantation, 24 Oct 2011. www.clinicaltrials.gov//ct2/show/NCT01459107 (2012). Accessed 4 Aug 2012.

20. Schneeberger S, Gorantla VS, Brandacher G, et al. Upperextremity transplantation using a cell-based protocol to minimize immunosuppression. Ann Surg. 2012. doi:10.1097/SLA.0b013e $31826 \mathrm{~d} 90 \mathrm{bb}$

21. - Bonastre J, Landin L, Diez J, et al. Factors influencing acute rejection of human hand allografts: a systematic review. Ann Plast Surg. 2012;68(6):624-9. This reports shows factors that may trigger rejection in hand transplantation.

22. Petruzzo P, Kanitakis J, Badet L, et al. Long-term follow-up in composite tissue allotransplantation: in-depth study of five (hand and face) recipients. Am J Transpl. 2011;11(4):808-16.

23. - Lantieri L, Hivelin M, Audard V, et al. Feasibility, reproducibility, risks and benefits of face transplantation: a prospective study of outcomes. Am J Transpl. 2011;11(2):367-78. This report summarizes the largest clinical series of face transplants.

24. Cavadas PC, Ibanez J, Thione A. Surgical aspects of a lower face, mandible, and tongue allotransplantation. J Reconstr Microsurg. 2012;28(1):43-7.

25. Pomahac B, Pribaz J, Eriksson E, et al. Three patients with full facial transplantation. N Engl J Med. 2012;366(8):715-22.

26. Glaus SW, Johnson PJ, Mackinnon SE. Clinical strategies to enhance nerve regeneration in composite tissue allotransplantation. Hand Clin. 2011;27(4):495-509.

27. Phan DQ, Schuind F. Tolerance and effects of FK506 (tacrolimus) on nerve regeneration: a pilot study. J Hand Surg Eur Vol. 2012;37(6):537-43.

28. Schneeberger S, Gorantla VS, Hautz T, et al. Immunosuppression and rejection in human hand transplantation. Transpl Proc. 2009;41(2):472-5.

29. Schneeberger S, Gorantla VS, van Riet RP, et al. Atypical acute rejection after hand transplantation. Am J Transpl. 2008;8(3): 688-96.
30. Landin L, Cavadas PC, Ibanez $\mathrm{J}$, et al. $\mathrm{CD}^{+}$-mediated rejection and $\mathrm{C} 4 \mathrm{~d}$ deposition in two composite tissue (bilateral hand) allograft recipients after induction with alemtuzumab. Transplantation. 2009;87(5):776-81.

31. Kaufman CL, Breidenbach W. World experience after more than a decade of clinical hand transplantation: update from the Louisville hand transplant program. Hand Clin. 2011;27(4):417-21, vii-viii.

32. - Kaufman CL, Ouseph R, Blair B, et al. Graft vasculopathy in clinical hand transplantation. Am J Transpl. 2012;12(4):1004-16. This is the first manuscript reporting chronic allograft rejection.

33. Unadkat JV, Schneeberger S, Horibe EH, et al. Composite tissue vasculopathy and degeneration following multiple episodes of acute rejection in reconstructive transplantation. Am J Transpl. 2010;10(2):251-61.

34. Kanitakis J, McGregor B, Badet L, et al. Absence of c 4 d deposition in human composite tissue (hands and face) allograft biopsies: an immunoperoxidase study. Transplantation. 2007;84(2):265-7.

35. Petruzzo P, Dubernard JM. World experience after more than a decade of clinical hand transplantation: update on the French program. Hand Clin. 2011;27(4):411-6, vii.

36. Jablecki J. World experience after more than a decade of clinical hand transplantation: update on the Polish program. Hand Clin. 2011;27(4):433-42, viii.

37. Gordon CR, Siemionow M, Papay F, et al. The world's experience with facial transplantation: what have we learned thus far? Ann Plast Surg. 2009;63(5):572-8.

38. - Gordon CR, Abouhassan W, Avery RK. What is the true significance of donor-related cytomegalovirus transmission in the setting of facial composite tissue allotransplantation? Transpl Proc. 2011;43(9):3516-20. This report reviews current knowledge of CMV infection.

39. - Gordon CR, Avery RK, Abouhassan W, et al. Cytomegalovirus and other infectious issues related to face transplantation: specific considerations, lessons learned, and future recommendations. Plast Reconstr Surg. 2011;127(4):1515-23. This report reviews current knowledge of post-transplant infections.

40. Bonatti H, Aigner F, De Clercq E, et al. Local administration of cidofovir for human papilloma virus associated skin lesions in transplant recipients. Transpl Int. 2007;20(3):238-46.

41. Reilly KE, Linz JC, Stern PJ, et al. Osteomyelitis of the tubular bones of the hand. J Hand Surg Am. 1997;22(4):644-9.

42. Bonatti H, Lass-Florl C, Zelger B, et al. Alternaria alternata soft tissue infection in a forearm transplant recipient. Surg Infect (Larchmt). 2007;8(5):539-44.

43. Landin L, Cavadas PC, Garcia-Cosmes P, et al. Perioperative ischemic injury and fibrotic degeneration of muscle in a forearm allograft: functional follow-up at 32 months post transplantation. Ann Plast Surg. 2011;66(2):202-9. 